author version: JOURNAL OF MASS SPECTROMETRY (ISSN: 1076-5174) (eISSN: 1096-9888) 50: (10) pp. 1130-1135. (2015)

DOI: $10.1002 / \mathrm{jms} .3629$

\title{
Simple correction improving long-term reproducibility of HPLC-MS
}

Eszter Tóth ${ }^{1,2}$, Helga Hevér ${ }^{3,4}$, Olivér Ozohanics ${ }^{1,5}$, András Telekes ${ }^{6}$, Károly Vékey ${ }^{1,7}$ and László Drahos ${ }^{1,5}$

${ }^{1}$ MS Proteomics Research Group, Research Centre for Natural Sciences, Hungarian Academy of Sciences, H-1519 Budapest, P.O. Box 286., Hungary

${ }^{2}$ Semmelweis University, School of Ph.D. Studies, Üllöi út 26., H-1085 Budapest, Hungary

${ }^{3}$ Gedeon Richter PLC, H-1475 Budapest, P.O. Box 27., Hungary

${ }^{4}$ Department of Inorganic and Analytical Chemistry, Budapest University of Technology and Economics, Szent Gellért tér 4., H-1111 Budapest, Hungary

${ }^{5}$ MTA-TTK NAP B MS Neuroproteomics Research Group, Research Centre for Natural Sciences, Hungarian Academy of Sciences, H-1519 Budapest, P.O. Box 286., Hungary

${ }^{6}$ Department of Oncology, Bajcsy-Zsilinszky Municipal Hospital, Maglódi út 89., H-1106 Budapest, Hungary

${ }^{7}$ Core Technologies Centre, Research Centre for Natural Sciences, Hungarian Academy of Sciences, H-1519 Budapest, P.O. Box 286., Hungary

\section{Summary}

Chromatographic peak areas in long series of HPLC-MS experiments often vary, which decreases reproducibility and may cause bias in the results. It was found that the sensitivitiy of various components change differently; in our case variability is in the order of 20-40\%; and it is most likely due to changing conditions in ESI ionization. The most often used peak area correction methods do not take this effect into account. The change in peak areas can be well described by a polynomial function; we found that a $4^{\text {th }}$ order polynomial is most often suitable. We suggest a simple correction algorithm based on polynomial fitting. When the experiments were inherently well reproducible, this correction improved reproducibility from $12 \%$ to $3 \%$ (on average for various components). When random errors were larger, this improvement was less significant (15\% to $12 \%$ in nano-ESI), but nevertheless essential in order to avoid possible bias in the results.

Keywords: HPLC-MS, Electrospray, long-term stability, signal drift, proteomics 
author version: JOURNAL OF MASS SPECTROMETRY (ISSN: 1076-5174) (eISSN: 1096-9888) 50: (10) pp. 1130-1135. (2015)

DOI: $10.1002 / \mathrm{jms} .3629$

\section{Introduction}

Various mainstream applications rely on long series of experiments using electrospray ionization (ESI ${ }^{1}$ ), typically with HPLC-MS(MS). Proteomics, metabolomics, pharmaceutical analysis and environmental monitoring are typical application fields needing long experimental sequences. ESI, and in particular nano-ESI, is known to have issues with long term stability ${ }^{2}$ and achieving long term stability is still an analytical challenge. There are many known (such as ion suppression, MS performance drift due to contamination of the ion source, ageing of the analytical column, etc.) and also unknown effects and parameters, which influence peak area, fragmentation and redox processes occurring in ESI. Among these spraying mode is an important, and well-studied effect ${ }^{3,4}$. Poor reproducibility due to unstable ESI conditions hinders quantitation (or semiquantitation), although the use of multiple internal standards ${ }^{5}$ or isotope-labelled methods (such as isotope coded affinity $\operatorname{tags}^{6}$ or universal metabolome-standard method ${ }^{7}$ in metabolomics) offer excellent possibilities in this regard. However, application of isotope labelling is often not possible (or not feasible).

There are various attempts to improve stability/reproducibility of (nano)HPLC-(nano)ESI$\mathrm{MS}^{8-10}$; in particular in the field of metabolomics ${ }^{11-15}$. One of the most often used method is to use internal standards (IS). By selecting an internal standard similar to the studied compound, systematic batch-to-batch differences can be compensated. The chemicals of a very complex matrix, however, coeluting with the internal standard can cause ion suppression and therefore add systematic bias ${ }^{5}$. A further limitation, especially in the field of proteomics, is the lack of isotope labelled internal standards for most peptides and even more for modified peptides (like glycopeptides). Another alternative is to use the total intensity of the peaks; Wang at al. used total abundance regression calibration method to compensate for injection and other effects ${ }^{13}$. A significant limitation of methods using the total intensity of the peaks is, that it does not take into account the effect of individual peaks. If abundance of some peaks are increased while others are decreased these methods cannot correct it. In a recent publication Kuligowski ${ }^{16}$ used $\delta$ statistics to detect batch effect in large data sets, but they did not propose a correction method. A popular alternative is to measure QC samples among the samples and use the intensity of QC peaks for correction $^{14,15}$.

In the present paper we address the issue of repeatability and reproducibility in long series of HPLC-MS experiments using both conventional and nano-electrospray. We focus on cases when relatively small differences among similar samples are studied: This is the case for most biomarker discovery studies, like identifying differences among plasma samples; or quality control of pharmaceutical products. Examples in proteomics and in pharmaceutical applications will be discussed. 
DOI: $10.1002 / \mathrm{jms} .3629$

\section{Experimental}

\section{Samples and chemicals}

Human blood plasma sample was obtained from a healthy volunteer from BajcsyZsilinszky Hospital, Budapest, Hungary (ethical permission number 1031-6/2012). The infliximab (Remicade) sample was obtained from Janssen Biotech (USA). 1,4-dithio-L,Dthreitol (DTT) and 2-iodoacetamide (IAA) were obtained from Fluka Chemie GmbH (Sigma-Aldrich $®$, Zwijndrecht, Netherlands). RapiGest SF (lyophilized sodium-3-[(2methyl-2-undecyl-1,3-dioxolan-4-yl)-methoxyl]-1-propane-sulfonate) was purchased from Waters (Milford, MA, USA). Mass spectrometry grade trypsin (Promega Corporation, Madison, WI, USA) was used. All other reagents were purchased from Sigma-Aldrich ${ }^{\circledR}$ (St. Louis, MO, USA).

\section{Sample preparation}

Plasma samples were depleted for albumin and $\operatorname{IgG}$ using Agilent Multiple Affinity Removal Spin Cartridge HSA/IgG (Agilent Technologies, Santa Clara, CA, USA), according the manufacturer's standard protocol. Enzymatic digestion of depleted plasma samples and infliximab were performed using trypsin, according to the protocol described before $^{17,18}$.

\section{LC-MS analysis}

HPLC-MS analysis of the infliximab digest was performed on a Nexera UPLC (Shimadzu Corporation) coupled to a high resolution micrOTOF-Q II mass spectrometer (Bruker Corporation). Chromatographic conditions were the following: the analytical column was a Kinetex reversed-phase column $(1.7 \mu \mathrm{m}$ XB-C18 particles, $2.1 \mathrm{~mm}$ i.d. $\times 100 \mathrm{~mm}$, Phenomenex Inc., CA, USA). Solvent A was water containing $0.1 \mathrm{v} / \mathrm{v} \%$ formic acid and solvent B was $10 \%$ water and $90 \%$ acetonitrile containing $0.1 \mathrm{v} / \mathrm{v} \%$ formic acid. The gradient was using $200 \mu \mathrm{L} / \mathrm{min}$ flow rate starting with a $8 \mathrm{~min}$ isocratic period with $3 \% \mathrm{~B}$, then a 50 min long gradient going to $50 \%$ solvent B. Washing was done using 7 min long gradient from 50 to $100 \%$ solvent B, and kept there for $4 \mathrm{~min}$. After that returning to $3 \%$ B in $1 \mathrm{~min}$, and equilibration was done for $10 \mathrm{~min}$. The column temperature was $30^{\circ} \mathrm{C}$. Mass spectrometric conditions: The mass spectrometer was used in positive electrospray ionization mode. Capillary voltage was $4.5 \mathrm{kV}$, nebulizer gas was 1 bar, dry gas was 100 $\mathrm{L} / \mathrm{min}$, dry temperature $200^{\circ} \mathrm{C}$, end plate offset was $500 \mathrm{~V}$. Scans were acquired in the 80 $2200 \mathrm{~m} / \mathrm{z}$ range.

HPLC-MS analysis of the depleted plasma digest was performed on a nanoAcquity UPLC (Waters, Milford, MA, USA) coupled to a high resolution QTOF Premier mass spectrometer (Waters, Milford, MA, USA). Chromatographic conditions were the following: A Symmetry C18 trap column $(180 \mu \mathrm{m}$ i.d. $\times 20 \mathrm{~mm}$, Waters Milford, MA, USA) was used, the analytical column was a reversed-phase column (C18, $1.7 \mu \mathrm{m} \mathrm{BEH}$ particles, $75 \mu \mathrm{m}$ i.d. $\times 200 \mathrm{~mm}$, Waters, Milford, MA, USA). Solvent A was water containing $0.1 \mathrm{v} / \mathrm{v} \%$ formic acid and solvent $B$ was acetonitrile containing $0.1 \mathrm{v} / \mathrm{v} \%$ formic acid. The gradient was using $250 \mathrm{~nL} / \mathrm{min}$ flow rate starting with a 4 min gradient from $3 \%$ 
DOI: $10.1002 / \mathrm{jms} .3629$

to $8 \% \mathrm{~B}$, then a $65 \mathrm{~min}$ long gradient going to $40 \%$ solvent $\mathrm{B}$. Washing was done using $450 \mathrm{~nL} / \mathrm{min}$ flow rate and a $2 \mathrm{~min}$ long gradient from 40 to $75 \%$ solvent $\mathrm{B}$, and kept there for $18 \mathrm{~min}$. After that returning to $3 \% \mathrm{~B}$ in $2 \mathrm{~min}$, and equilibration was done for $18 \mathrm{~min}$. The column temperature was $55^{\circ} \mathrm{C}$. Mass spectrometric conditions: The mass spectrometer was used in positive electrospray ionization mode. Capillary voltage was $2.3 \mathrm{kV}$, nanoflow 1 bar, source temperature $90{ }^{\circ} \mathrm{C}$, cone voltage $35 \mathrm{~V}$. Single stage mass spectrometry in extended dynamic range mode was applied. Scans were acquired in the $500-2000 \mathrm{~m} / \mathrm{z}$ range.

\section{Data evaluation}

In all cases chromatographic peak areas were measured, based on the most abundant isotope of the most abundant charge state of the protonated molecule (e.g. $[\mathrm{M}+3 \mathrm{H}] 3+)$. In the Bruker instrument DataAnalysis Version 4.0 SP2 program was used for to determine peak areas. In the case of the Waters instrument an in-house developed software, GlycoPattern v.3.0 ${ }^{17}$ was used to determine peak areas. The $4^{\text {th }}$ order polynomial fitting described in the text was performed using least squares minimization algorithm. Polynomial fitting and calculations based on Eq. 1 were facilitated by a VBA macro incorporated in an Excel worksheet. This Excel file is available from the authors on request.

\section{Results and discussion}

The first experiment to be discussed is analysis of a series of tryptic digests of infliximab, a pharmaceutical monoclonal antibody $(\mathrm{mAb})$. The objective was to find small changes in the peptide digest due to sample storage at various temperatures. The digests were studied using UHPLC-ESI-MS on a Bruker QTOF instrument. The chromatograms were $80 \mathrm{~min}$ long; altogether 57 HPLC-MS runs were performed in the course of a 4 day long experiment (see details in the Experimental section). The relative peak area of 18, randomly chosen components in the sample was evaluated; both major and minor peaks, eluting at various retention times; among them both peptides and glycopeptides. Chromatographic peak areas were calculated from the monitored signals of the most abundant isotopic peak of the protonated molecule in its most abundant charge state. Peak areas were normalized to their average area in the chromatographic series.

In the course of the experimental sequence a reference compound (the same digest, stored at $-20 \mathrm{C}$ ) was injected 13 times, approximately randomly distributed among the samples. The reproducibility/variability of various components in the reference sample were evaluated to assess the stability of HPLC-ESI-MS experiments, using peak areas of the 18 selected components described above. Variability among these is characterized by the relative standard deviation; which is conventional. Similarity between the set of peak areas in two selected chromatograms may be compared by various methods; among these we have chosen the Pearson correlation coefficient; which is frequently used. 
DOI: $10.1002 / \mathrm{jms} .3629$

Average relative standard deviation of peak areas, measured in absolute (arbitrary) units is 12\%. Relative peak areas and their standard deviations are listed in Table 1. Most peak areas increase with time (in the sequence of the injections); but there are peaks which decrease in area along the injection series. Three such examples are shown in Fig. 1. This Figure clearly shows that there is an obvious time-dependence of the data; but the time dependence is different for the various components. Note, all of the components studied are protonated peptides; i.e. they are chemically similar. Behavior of various components might be expected to be even more different if different compound types were studied. Note that no obvious correlation have been found between the properties of peptides (peptide size, sequence or polarity) and their changes of peak areas in a series of chromatograms.

(a) Experimental data

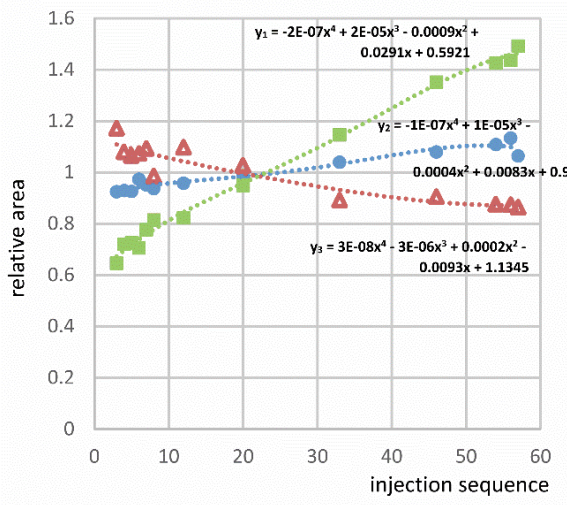

(b) Time-corrected data

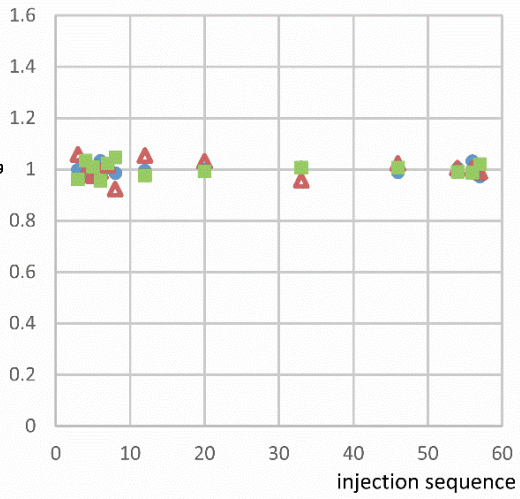

Fig. 1: Changes of peak areas in a series of chromatograms of infliximab digest, as a function of the injection sequence. Three chromatographic peaks were selected in the infliximab digest (Peaks \# 4, 5 and 12 in Table 1). Peak areas were normalized to their average area in the series: (a) experimental data; (b) results after correction of time dependence. Experimental peak areas vary significantly in the experimental sequence (and each peak in a different manner), while only small random errors are present after time-correction. See details in text. 
DOI: $10.1002 / \mathrm{jms} .3629$

The similarity between two individual chromatograms is often characterized by the Pearson correlation coefficient ( $R$; note that often the square of this number, $\mathrm{R}^{2}$ is specified $)^{19}$. These coefficients were calculated for all combinations of the chromatograms (13 chromatograms yield altogether 78 such values). These values are arranged in Fig. 2 as a function of the time difference between the chromatograms (blue open circles). The correlation coefficient shows good overall similarities (the $\mathrm{R}$ value is well above 0.99 in all cases). However, the correlation coefficient decreases significantly with increasing time between individual sample injections. In other words, when the time between individual chromatograms is short, these are similar; but when long time has elapsed between two chromatographic runs, these start to become different.

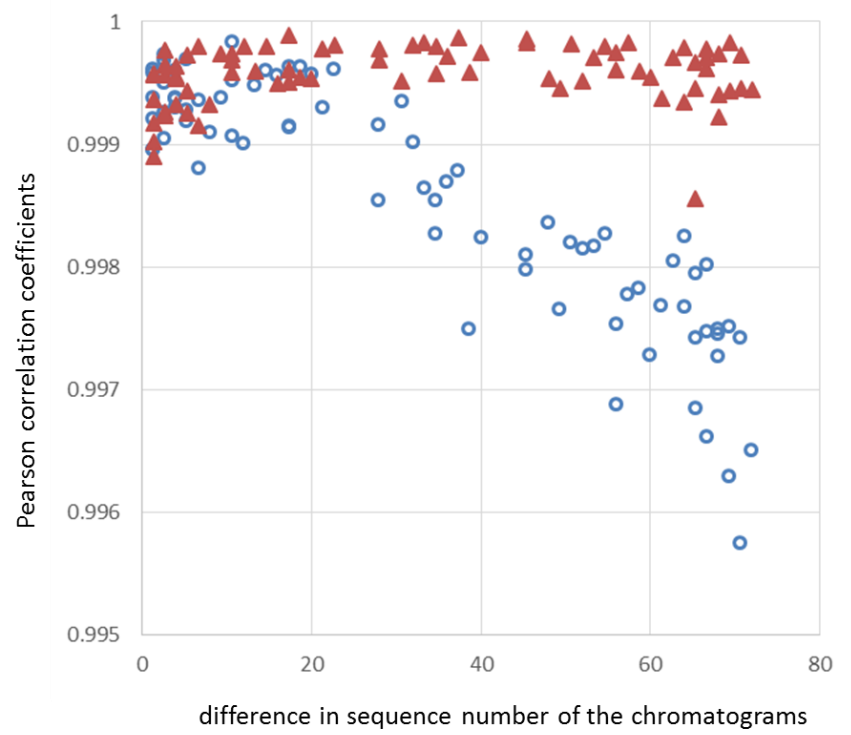

Fig. 2: Pearson correlation coefficients $(\mathrm{R})$ between various pairs of reference chromatograms in a series of infliximab digest, shown as a function of the timedifference between the individual injections (measured by difference in sequence number of the chromatograms). Blue open circles represent original data; red triangles show the result after correcting peak areas for their time-dependence. The Figure shows that the difference between pairs of chromatograms increases, when the time between them increases; but this can be well compensated. 
DOI: $10.1002 / \mathrm{jms} .3629$

Figs. 1 and 2 suggest that the major source of variability of peak areas (as measured by the relative standard deviations in Table 1) is due to the time-dependence of the experiments, and not due to random errors. It follows, that taking into account the time dependence of chromatograms (i.e. that of changes in mass spectrometer conditions) this error might be compensated. This would have three major consequences: (1) This would improve reproducibility and repeatability (measured by relative standard deviation); (2) eliminate a major source for "bias" (e.g. mistakenly evaluating time-dependence as a certain biological/chemical effect); and (3) may help standardizing experiments run on different instruments or in different laboratories. Note that time dependence of each individual peak (identified by retention time and $\mathrm{m} / \mathrm{z}$ value) should be considered separately; as they may change differently in time (Fig.1).

In order to compensate for time-dependence, a reference sample should be measured at various times in the experimental sequence. The reference samples should be similar to the samples studied (should contain the same components, although the abundance of these components in not essential). Ideally a pool of the samples to be studied should be used; although samples from a control experiment may be equally suitable. Conventional quality control (QC) samples (like a synthetic peptide mixture, or an enolase digest) may not help in this respect, as sensitivity of various compounds changes differently.

We suggest a simple algorithm to correct the time-dependence (changing MS conditions) discussed above. We measure the peak area of a selected ion signal in the reference sample (like that in Fig. 1); and describe its time dependence with a polynomial (linear or quadratic would be well suited for those shown in Fig. 3). Parameters of the polynomial were determined by least squares fitting. Subsequently we correct the measured peak areas (both in the case of reference and "real" samples) according to the following equation:

$$
A_{x}^{\text {corr }}=A_{x}^{\text {meas }} * \frac{A_{\text {avr }}}{f(x)}
$$

Where $\mathrm{A}_{\mathrm{x}}{ }^{\text {corr }}$ is the corrected peak area of the selected ion at the $\mathrm{x}$-th chromatogram in the series; $\mathrm{A}_{\mathrm{x}}$ meas is the measured peak area in the $\mathrm{x}$-th chromatogram; $\mathrm{A}_{\mathrm{avr}}$ is the averaged peak area in the series of reference compound measurements; and $f(x)$ is the expected peak area of the reference compound if it were measured in the $x$-th chromatogram, based on the fitted polynomial function. Note that, in our case retention time shifts are small, on average $0.4 \%$, therefore no corrections were necessary.

Data in Fig. 1 could be well approximated by a linear function, but the time dependence is not always so straightforward. We have studied a number of chromatogram series (the instrument is never tuned during a given series of chromatographic runs); and some other examples will be discussed below. In our experience the change in signal dependence could be well fitted to a $4^{\text {th }}$ power function in all cases. If there are more than ca. 10-12 reference 
DOI: $10.1002 / \mathrm{jms} .3629$

chromatograms studied, there is no serious problems with over-fitting. Using the $4^{\text {th }}$ order polynomial function with Equation 1 (the fitted polynomial curves are shown in Fig. 1), we have corrected the measured peak areas in the experiment described above. Taking into account time-dependence using this simple correction algorithm decreases the average relative standard deviation of peak areas from $12 \%$ to $3 \%$ (Table 1). We have also compared the similarity of various chromatograms using the Pearson correlation coefficients, as described above. The correlation coefficients improve significantly, and are better than 0.999 in all cases, even when several days elapse between injections. The data after time-correction are shown by the red triangles in Fig. 2. These results indicate that the time dependence (i.e. variability of mass spectrometer conditions) has clearly been well-compensated by the simple algorithm suggested.

A different, experimentally more demanding example is encountered in the case of glycosylation analysis of plasma proteins. Tryptic digests of a depleted plasma sample were measured and the glycosylation patterns were studied based on 16 glycopeptide peak areas (like before, based on relative chromatographic peak area of the most abundant isotope of the protonated molecule in the most abundant charge state). These experiments have been performed on a nano-UHPLC on a Waters QTOF instrument. Altogether 52, individually 120 min long chromatograms were studied, the objective was to determine reproducibility of various sample preparation steps. Sample preparation may result in sample loss; which was compensated by normalizing the results (set of selected ion peak areas) to the sum of the 16 peak areas studied. Partly due to the use of nano-ESI (which is known to be much less robust than conventional ESI); partly due to random errors in sample preparation; and partly because many of the studied peaks were close to the detection threshold, the overall scattering of the data is significantly larger than that discussed above. The results in one example (the biantennary, twice sialylated glycoform of the MVSHHNLTTGATLINEQWLLTTAK peptide derived from haptoglobin) are shown in Fig. 3. The uncorrected results (blue circles) show significant time-dependence; which can be well represented by a $4^{\text {th }}$ order polynomial (dotted line). Time dependence, as before, is different for the various ions considered. Correcting the data for time-dependence (using Eq. 1) results in the red triangles in Fig. 3. Although the scattering of data is significant (especially compared to Fig. 1); the time dependence is well taken care of. In this case the average relative standard deviation of uncorrected peak areas is $15 \%$; time correction reduces this to $12 \%$. This improvement is much less striking, than those discussed in the previous case; mainly due to large overall random errors. Nevertheless, systematic errors can be compensated this way, and that may be very important for avoiding bias. Note that in this case systematic errors (those, which are nearly completely removed by the correction) are of similar size than random errors; the relatively small improvement is due to the laws of error propagation. 
author version: JOURNAL OF MASS SPECTROMETRY (ISSN: 1076-5174) (eISSN: 1096-9888) 50: (10) pp. 1130-1135. (2015)

DOI: $10.1002 / \mathrm{jms} .3629$

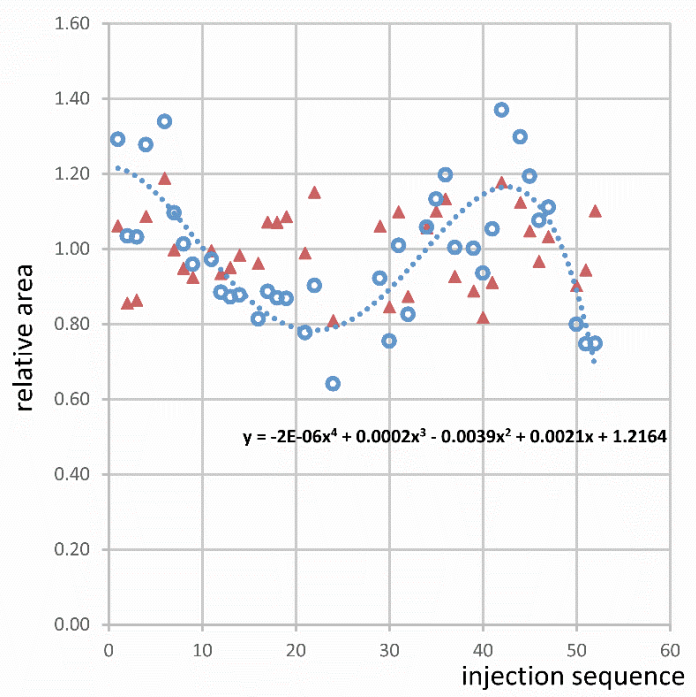

Fig. 3: Changes of relative peak areas in a series of chromatograms of all plasma digests. The selected peak is the biantennary, twice sialylated glycoform of the MVSHHNLTTGATLINEQWLLTTAK peptide derived from haptoglobin. Blue open circles indicate measured peak areas; red triangles those after timecorrection. The blue dots show the $4^{\text {th }}$ order polynomial used to describe timedependence. The results are plotted as a function of injection sequence. Random errors are significant even after time-correction, but the systematic change in peak areas (which may cause bias in the results) is removed.

In the paragraphs above, we have shown time dependence (and its correction) for the main charge state of various compounds. We have checked other charge states as well, and found that various charge states of the same compound show different evolution in time. This suggests, that time dependence is predominantly due to changes in mass spectrometric (electrospray) conditions. In some cases increase in the peak area of e.g. 3+ state was accompanied with decrease in the peak area of the $2+$ charge state, (one such example is shown in Fig. 4, which illustrates the (absolute, uncorrected) peak areas of \#6 VYACEVTHQGLSSPVTK peptide in Table 1) but our data do not suggest that this could be generalized. When necessary, the various charge states could be treated (corrected) separately. For relative quantitation (e.g. amount of the analyte in sample \#1 compared to sample \#2) measuring the peak areas of any charge state (or the sum of all charge states) is equally suitable. Absolute quantitation (provided that suitable, preferably isotope labeled 


\section{DOI: $10.1002 / \mathrm{jms} .3629$}

internal standard is available) can also be based on the peak area of any charge state. For this reason, for analytical purposes, it is sufficient to characterize (and correct) the time behaviour of an arbitrarily selected charge state only.

In Figs. 1-3 we have shown relative peak areas; as we believe those are most useful for analytical purposes. Normalizing data to the sum of all peak areas is common practice. This removes some of the errors in sample preparation and sample injection; which errors are independent of the time-dependence studied here, and may be important in practice. Normalization also removes errors due to long-term change in overall mass spectrometric sensitivity. This may be combined with the $4^{\text {th }}$ degree polynomial fitting suggested in the present paper. We have checked, that (when there are no errors due to sample preparation) the results of the $4^{\text {th }}$ degree polynomial fitting with or without normalization are very similar. Overall sensitivity in long series of MS measurements often shows a decreasing trend (i.e. decreasing MS performance). However, it is not always the case. The experiment series discussed in Figs. 1, 2 and 4, for example, shows an increasing trend in overall sensitivity (Fig. 4, middle curve).

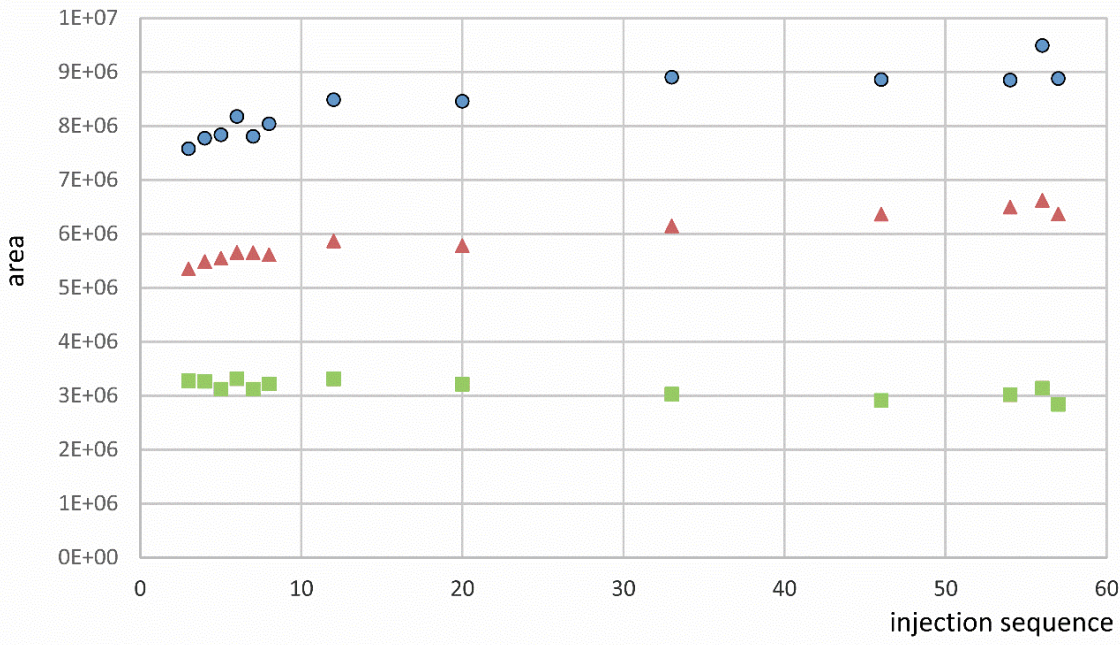

Fig. 4: Changes of peak areas of infliximab in a series of chromatograms, as a function of the injection sequence. Different charge states of VYACEVTHQGLSSPVTK peptide (\#6 in Table 1) are represented using blue dots: $\mathrm{m} / \mathrm{z} 625.983+$; and green squares: $\mathrm{m} / \mathrm{z} 938.47$ $2+$. The data show that when peak area of $3+$ charge state increases, then area of $2+$ 
DOI: $10.1002 / \mathrm{jms} .3629$

decreases. Red triangles indicates the sum of peak areas of all peptides (divided by 10) showing that overall sensitivity increases.

\section{Conclusions}

Long experimental sequences, lasting several days or more are becoming common practice in HPLC-MS, especially in proteomics, in metabolomics and in pharmaceutical quality control. The experimental conditions may subtly change during this time, which may result in a systematic change of relative peak areas. This effect was illustrated in two cases: (a) looking at various peptides in an infliximab digest, using a conventional ESI source; and (b) glycopeptides in a human plasma digest using nano-ESI ion source; on a Bruker and a Waters instrument, respectively. Both experiments lasted 3-4 days, and chromatographic peak areas (measured in selected ion chromatograms) of various components were monitored. Evaluation of the results showed that:

a) Peak areas vary in time; this may be close to linear (Fig. 1) or may be more complex (Fig. 3). In the present example the systematic change was in the order of $20-40 \%$ for most components (resulting in 10-20\% relative standard deviation). Results obtained with a conventional and a nano-ESI source were comparable in this respect. The similarity between individual chromatograms decreases when the time between the chromatograms increases (Fig. 2). The most likely reason for the timedependence is changing conditions in ESI ionization. When tuning in the ion source was intentionally changed (like capillary voltage, nebulizer pressure, etc.), this resulted in a similar difference in peak areas as that observed in the course of several days.

b) The change of peak areas in time is different for the various components (Fig. 1). Here only protonated molecules were studied; the change in the case of fragment ions or molecular adducts may be even more significant. Note that most existing peak area correction methods do not take this into account and may lead to significant bias.

c) The time dependence can be described in all cases studied by a $4^{\text {th }}$ order polynomial function (this includes various examples in our laboratory, not only those described in the present paper). A simple correction function (Eq 1) is suggested, which removes the time-dependence from the data. When random errors are small, this leads to a dramatic improvement of reproducibility (12 to $3 \%$ rsd, Fig. 1). When random errors are large, improvement in reproducibility is modest (15 to $12 \%$ rsd, Fig. 3). Nevertheless, correction is important in this case as well, as it removes a major source for bias in the experiment sequence.

d) Correcting the time dependence of the data relies on the use of a reference compound; which should be injected various times during the experiment sequence. The reference sample should be similar and should contain all components monitored in the sample. Ideally it is expedient to inject a reference sample after 3- 


\section{DOI: $10.1002 / \mathrm{jms} .3629$}

5 samples. If a $4^{\text {th }}$ order polynomial function is used to describe time dependence, at least 10-15 reference samples should be run, to avoid over-fitting the parameters.

e) The same reference sample may be used to improve comparison of different series of HPLC-MS runs; like experimental series running for several month or improve inter-laboratory comparisons. For each monitored compound the average peak area in the reference sample (in a given HPLC-MS series) is measured, and is compared to that measured in a different HPLC-MS series $\left(\mathrm{A}^{\text {ref }}(1)\right.$ and $\mathrm{A}^{\mathrm{ref}}(2)$, respectively). The ratio of the two values characterizes the relative sensitivity (for the selected compound) in the two experiment series. To make sample peak areas comparable in the second series to that observed in the first series, the following equation may be used:

$$
A^{\text {mod }}=A^{\text {meas }}(2) * \frac{A^{r e f}(1)}{A^{r e f}(2)}
$$

Where $\mathrm{A}^{\text {meas }}(2)$ is the measured (time-corrected) peak area of a selected component in a sample measured in the \# $2^{\text {nd }}$ series of experiments; while $\mathrm{A}^{\text {mod }}$ is its modified value comparable in scale with experiments in a different series (or in a different laboratory).

When similar samples are studied, the suggested method may be applied without using quality control samples; although the correction will be less accurate. In such a case (for the purpose of correcting instrumental variability) all samples are considered being identical (as if all were QC samples). Correction (using a polynomial) is performed, as described above. After correction, the remaining variability in the data set is equated with the biological variability among the samples. Note that in this case it is particularly important that samples should be measured in a random order; and that correction will be less reliable. Our experience suggests that without QC samples 50-70\% of the instrumental variability may be expected to be removed this way.

\section{Acknowledgement}

The authors are indebted for financial support of Hungarian Scientific Research Fund OTKA NK83857 and OTKA K109006; National Brain Research Program (NAP) KTIA_NAP_12-2-2015-0003, Research and Technology Innovation Fund VKSZ-12-12013-0001. We thank the Core Technology Centre of the Research Centre for Natural Sciences of the Hungarian Academy of Sciences for the use of the Waters HPLC-MS instrument. We also thank Ibolya Kreutzne Kun (Gedeon Richter PLC, Spectroscopic Research Department) for her technical assistance. We gratefully acknowledge Dr Csaba Szantay Jr (Gedeon Richter PLC, Spectroscopic Research Department) for his professional support.

\section{References:}




\section{DOI: $10.1002 / \mathrm{jms} .3629$}

[1] J.B. Fenn. Electrospray wings for molecular elephants (Nobel lecture). Angewandte Chemie - International Edition 2003, 42, 3871-3894.

[2] N.B. Cech, C.G. Enke. Practical implications of some recent studies in electrospray ionization fundamentals. Mass Spectrom Rev 2001, 20, 362-87.

[3] P. Nemes, I. Marginean, A. Vertes. Spraying Mode Effect on Droplet Formation and Ion Chemistry in Electrosprays. Analytical Chemistry 2007, 79, 3105-3116.

[4] P. Nemes, S. Goyal, A. Vertes. Conformational and Noncovalent Complexation Changes in Proteins during Electrospray Ionization. Analytical Chemistry 2007, 80, 387-395.

[5] H. Redestig, A. Fukushima, H. Stenlund, T. Moritz, M. Arita, K. Saito, M. Kusano. Compensation for Systematic Cross-Contribution Improves Normalization of Mass Spectrometry Based Metabolomics Data. Analytical Chemistry 2009, 81, 7974-7980.

[6] S.P. Gygi, B. Rist, S.A. Gerber, F. Turecek, M.H. Gelb, R. Aebersold. Quantitative analysis of complex protein mixtures using isotope-coded affinity tags. Nat Biotechnol 1999, 17, 994-9.

[7] J. Peng, Y.-T. Chen, C.-L. Chen, L. Li. Development of a Universal MetabolomeStandard Method for Long-Term LC-MS Metabolome Profiling and Its Application for Bladder Cancer Urine-Metabolite-Biomarker Discovery. Analytical Chemistry 2014, 86, 6540-6547.

[8] S. Nahnsen, C. Bielow, K. Reinert, O. Kohlbacher. Tools for Label-free Peptide Quantification(). Molecular \& Cellular Proteomics : MCP 2013, 12, 549-556.

[9] M. Sandin, J. Teleman, J. Malmström, F. Levander. Data processing methods and quality control strategies for label-free LC-MS protein quantification. Biochimica et Biophysica Acta (BBA) - Proteins and Proteomics 2014, 1844, 29-41.

[10] B.-J.M. Webb-Robertson, M.M. Matzke, J.M. Jacobs, J.G. Pounds, K.M. Waters. A statistical selection strategy for normalization procedures in LC-MS proteomics experiments through dataset-dependent ranking of normalization scaling factors. PROTEOMICS 2011, 11, 4736-4741.

[11] M. Brown, D.C. Wedge, R. Goodacre, D.B. Kell, P.N. Baker, L.C. Kenny, M.A. Mamas, L. Neyses, W.B. Dunn. Automated workflows for accurate mass-based putative metabolite identification in LC/MS-derived metabolomic datasets. Bioinformatics 2011, 27, 1108-1112.

[12] M.A. Kamleh, T.M.D. Ebbels, K. Spagou, P. Masson, E.J. Want. Optimizing the Use of Quality Control Samples for Signal Drift Correction in Large-Scale Urine Metabolic Profiling Studies. Analytical Chemistry 2012, 84, 2670-2677.

[13] S.Y. Wang, C.H. Kuo, Y.J. Tseng. Batch Normalizer: a fast total abundance regression calibration method to simultaneously adjust batch and injection order effects in liquid chromatography/time-of-flight mass spectrometry-based metabolomics data and comparison with current calibration methods. Anal Chem 2013, 85, 1037-46. 
author version: JOURNAL OF MASS SPECTROMETRY (ISSN: 1076-5174) (eISSN: 1096-9888) 50: (10) pp. 1130-1135. (2015)

DOI: $10.1002 / \mathrm{jms} .3629$

[14] E. Zelena et al. Development of a Robust and Repeatable UPLC-MS Method for the Long-Term Metabolomic Study of Human Serum. Analytical Chemistry 2009, 81, 1357-1364.

[15] W.B. Dunn et al. Procedures for large-scale metabolic profiling of serum and plasma using gas chromatography and liquid chromatography coupled to mass spectrometry. Nat. Protocols 2011, 6, 1060-1083.

[16] J. Kuligowski et al. Detection of batch effects in liquid chromatography-mass spectrometry metabolomic data using guided principal component analysis. Talanta 2014, 130, 442-8.

[17] O. Ozohanics, L. Turiák, A. Puerta, K. Vékey, L. Drahos. High-performance liquid chromatography coupled to mass spectrometry methodology for analyzing site-specific N-glycosylation patterns. Journal of Chromatography A 2012, 1259, 200-212.

[18] L. Turiak, O. Ozohanics, F. Marino, L. Drahos, K. Vekey. Digestion protocol for small protein amounts for nano-HPLC-MS(MS) analysis. Journal of Proteomics 2011, 74, 942-947.

[19] J. Li, D.B. Hibbert, S. Fuller, G. Vaughn. A comparative study of point-to-point algorithms for matching spectra. Chemometrics and Intelligent Laboratory Systems 2006, 82, 50-58. 